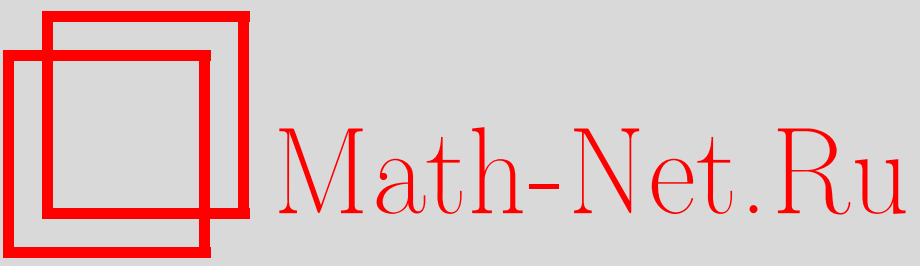

С. Руджинарс, Самосопряженные аналитические разностные операторы с нулевым отражением, ТМФ, 2001, том 128, номер 1, 116-132

DOI: https://doi.org/10.4213/tmf486

Использование Общероссийского математического портала Math-Net.Ru подразумевает, что вы прочитали и согласны с пользовательским соглашением

http://www . mathnet.ru/rus/agreement

Параметры загрузки:

IP : 54.237 .59 .107

26 апреля 2023 г., 10:50:30 
ТЕОРЕТИЧЕСКАЯ

И МАТЕМАТИЧЕСКАЯ

ФИЗИКА

Том 128, № 1

июль, 2001

(C) 2001 г.

\author{
С.Н.М. Руджинарс ${ }^{*}$
}

\title{
САМОСОПРЯЖЕННЫЕ АНАЛИТИЧЕСКИЕ РАЗНОСТНЫЕ ОПЕРАТОРЫ С НУЛЕВЫМ ОТРАЖЕНИЕМ
}

\begin{abstract}
Дается обзор работ автора по обыкновенным линейным аналитическим разностным операторам $(\mathrm{A} \Delta \mathrm{O})$ второго порядка, допускающим безотражательные собственные функции. Этот класс операторов гораздо шире, чем класс безотражательных операторов Шредингера и Якоби, соответствующих решетчатым солитонам Кортевегаде Фриза и Тоды. Показано, что подкласс безотражательных $\mathrm{A} \Delta \mathrm{O}$, обобщающий указанный класс дифференциальных и дискретных разностных операторов, соответствует солитонным решениям нелокальных эволюционных уравнений типа Тоды. Дальнейшие ограничения приводят к $\mathrm{A} \Delta \mathrm{O}$ с изометрическими преобразованиями собственных функций, которые можно использовать для связи самосопряженных операторов на $L^{2}(\mathbb{R}, d x)$ c A $\Delta \mathrm{O}$.
\end{abstract}

\section{1. ВВЕДЕНИЕ}

В работе [1] мы использовали полученные ранее результаты о безотражательных аналитических разностных операторах $(\mathrm{A} \Delta \mathrm{O})$ релятивистского типа КалоджероМозера [2], [3] как свидетельство в пользу предположения о сушествовании гораздо более широкого класса безотражательных $\mathrm{A} \Delta \mathrm{O}$. В настоящей работе мы обоснуем это предположение, а также дадим частичные ответы на связанные с ним вопросы о самосопряженности и о связанных солитонных эволюционных уравнениях. Подробности доказательств можно найти в серии последующих работ автора [3]-[6].

Начнем с простого наблюдения о “свободном" А $\Delta \mathrm{O}$

$$
A_{0} \equiv e^{-i \partial_{x}}+e^{i \partial_{x}},
$$

рассматриваемом как линейный оператор на пространстве $\mathcal{M}$ мероморфных функций. Очевидно, этот оператор имеет собственные функции $\mathcal{W}_{0}(x, \pm p)$ с собственным значением $e^{p}+e^{-p}$, где

$$
\mathcal{W}_{0}(x, p) \equiv e^{i x p}
$$

${ }^{*}$ Centre for Mathematics and Computer Science, Amsterdam, The Netherlands. E-mail: siru@wxs.nl 
Пусть теперь $\mu_{ \pm}(x, p)$ - функции, которые мероморфны и $i$-периодичны по $x$ при произвольном $p \in \mathbb{C}$. Тогда ясно, что

$$
\mathcal{W}_{0}^{\mu_{+}, \mu_{-}}(x, p) \equiv \mu_{+}(x, p) \mathcal{W}_{0}(x, p)+\mu_{-}(x, p) \mathcal{W}_{0}(x,-p)
$$

также является собственной функцией $A_{0}$ с собственным значением $e^{p}+e^{-p}$. Отсюда следует, что собственные пространства $\mathrm{A} \Delta \mathrm{O}$ второго порядка $A_{0}$ бесконечномерны, что находится в противоречии с двумерностью собственных пространств дифференциального оператора второго порядка $\partial_{x}^{2}$. Более того, свободу в выборе множителей можно использовать, чтобы построить бесконечномерные собственные пространства собственных функций в $L^{2}(\mathbb{R}, d x)$ (подобных $\left.\mathcal{W}_{0}(x, p) / \operatorname{ch} 2 \pi\left(x-x_{0}\right), x_{0} \in \mathbb{R}\right)$ и получить собственные функции любой заданной при $|\operatorname{Re} x| \rightarrow \infty$ плоско-волновой асимптотики. Например, полагая

$$
\mu_{-}(x, p) \equiv \frac{b(p) e^{-2 \pi x}}{e^{2 \pi x}+e^{-2 \pi x}}, \quad \mu_{+}(x, p) \equiv \frac{e^{2 \pi x}+a(p) e^{-2 \pi x}}{e^{2 \pi x}+e^{-2 \pi x}},
$$

легко проверить, что

$$
\mathcal{W}_{0}^{\mu_{+}, \mu_{-}}(x, p) \sim \begin{cases}e^{i x p}, & \operatorname{Re} x \rightarrow \infty \\ a(p) e^{i x p}+b(p) e^{-i x p}, & \operatorname{Re} x \rightarrow-\infty\end{cases}
$$

Пусть дана безотражательная асимптотическая функция, т.е. $b(p)=0$ и $|a(p)|=1$, $p \in \mathbb{R}$. Рассмотрим оператор в гильбертовом пространстве

$$
\mathcal{F}_{\mu_{+}}: \quad L^{2}(\mathbb{R}, d p) \rightarrow L^{2}(\mathbb{R}, d x), \quad f(p) \mapsto(2 \pi)^{-\frac{1}{2}} \int_{-\infty}^{\infty} d p f(p) \mu_{+}(x, p) e^{i x p} .
$$

Разумеется, если функция $\mu_{+}(x, p)$ определяется формулой $(1.2)$ и условием $a(p)=1$, этот оператор сводится к преобразованию Фурье $\mathcal{F}_{0}$. Поскольку преобразование Фурье $\mathcal{F}_{0}$ унитарно, по данному оператору $A_{0}$ получаем самосопряженный оператор $\hat{A}_{0}$ следующим образом:

$$
\hat{A}_{0} \equiv \mathcal{F}_{0} M \mathcal{F}_{0}^{-1},
$$

где $M$ - оператор умножения на $2 \operatorname{ch} p$ на пространстве $L^{2}(\mathbb{R}, d p)$. В исследуемом здесь свободном случае это дает естественный способ рассматривать $A_{0}$ как самосопряженньй оператор на $L^{2}(\mathbb{R}, d x)$.

Существуют бесконечномерное пространство мероморфных функций $a(p)$ таких, что $|a(p)|=1$ при вешественных $p$, и связанные с ними $i$-периодические мероморфные множители, удовлетворяющие условиям

$$
\mu(x, p) \sim \begin{cases}1, & \operatorname{Re} x \rightarrow \infty, \\ a(p), & \operatorname{Re} x \rightarrow-\infty,\end{cases}
$$

причем операторы $\mathcal{F}_{\mu}$, задаваемые формулой (1.3), также являются унитарными. Принимая это утверждение, получаем бесконечномерное пространство самосопряженных операторов

$$
\hat{A}_{\mu} \equiv \mathcal{F}_{\mu} M \mathcal{F}_{\mu}^{-1}
$$


связанных со свободным А $\Delta \mathrm{O} A_{0}$ на пространстве $\mathcal{M}$. Эти операторы можно сравнить с “очевидным" свободным оператором $\hat{A}_{0}$ в смысле теории рассеяния, и тогда, как и следовало ожидать, $S$-матрица является нетривиальной и задается, по существу, функцией $a(p)$.

Приведенные вьше рассуждения можно подтвердить результатами из раздела 5 работы [3]. В конце настоящей статьи мы приведем явньй пример только что описанного оператора $\hat{A}_{\mu}$ и, более того, подробно рассмотрим связь между нашим подходом и подходом работы [3]. Здесь же добавим только, что соответствуюшие множители $\mu(x, p)$ не имеют такого простого вида, как $\mu_{+}(x, p)$, задаваемый формулой (1.2). Действительно, используя развитые в работах [3], [6] средства, легко видеть, что для таких множителей оператор $\mathcal{F}_{\mu_{+}}$не изометричен (за исключением случая, когда $a(p)=1$, а потому $\left.\mathcal{F}_{\mu_{+}}=\mathcal{F}_{0}\right)$.

Мы начали с того, что привели некоторые факты, относящиеся к "свободному" выбору $A_{0}$, с тем, чтобы подготовиться к оценке соответствуюших свойств класса "взаимодействуюших" А $\Delta \mathrm{O}$ вида

$$
A \equiv e^{-i \partial_{x}}+V_{a}(x) e^{i \partial_{x}}+V_{b}(x)
$$

Здесь предполагается, что $V_{a}, V_{b} \in \mathcal{M}$ и

$$
\lim _{|\operatorname{Re} x| \rightarrow \infty} V_{a}(x)=1, \quad \lim _{|\operatorname{Re} x| \rightarrow \infty} V_{b}(x)=0 .
$$

Поэтому $A$ редуцируется к $A_{0}$ при $|\operatorname{Re} x| \rightarrow \infty$.

Первый естественный вопрос состоит в том, допускает ли $\mathrm{A} \Delta \mathrm{O} A$ собственные функции с собственным значением $e^{p}+e^{-p}$ и плоско-волновую асимптотику при $|\operatorname{Re} x| \rightarrow \infty$. Более точно, существуют ли функции $\mathcal{W}(\cdot, p) \in \mathcal{M}$, удовлетворяющие условиям

$$
\begin{gathered}
(A \mathcal{W})(x, p)=\left(e^{p}+e^{-p}\right) \mathcal{W}(x, p), \\
\mathcal{W}(x, p) \sim \begin{cases}e^{i x p}, & \operatorname{Re} x \rightarrow \infty, \\
a(p) e^{i x p}+b(p) e^{-i x p}, & \operatorname{Re} x \rightarrow-\infty,\end{cases}
\end{gathered}
$$

при произвольном $p \in \mathbb{C}$ ?

Из этого вопроса следует второй вопрос, являюшийся более сушественным для наших целей: можно ли найти безотражсательные функции $\mathcal{W}(x, p)$ с указанными свойствами (т.е. такие, что $b(p)=0$ в (1.9))? Подчеркнем сразу, что если такие функции можно найти, то функция $a(p)$ произвольна. Действительно, ее всегда можно изменить путем умножения $\mathcal{W}(x, p)$ на подходящие $i$-периодические функции $\mu(x, p)$

Наконец, третий вопрос: если $A$ допускает безотражательные собственные функции, то можно ли использовать их для того, чтобы связать самосопряженный оператор $\hat{A}$ на $L^{2}(\mathbb{R}, d x)$ с $A$ (подобно тому, как это показано для $\left.A_{0}\right)$ ? По нашему мнению, это важнейший функционально-аналитический вопрос. Действительно, в случаях, когда функция $\mathcal{W}(x, p)$ дает изометрическое преобразование собственных функций, изометрия обычно разрушается после умножения на нетривиальный множитель $\mu(x, p)$. 
Прежде чем приступить к подробному изучению этих общих вопросов, весьма полезно вспомнить, что для операторов Шредингера и Якоби можно получить полные ответы на второй и третий вопросы с помошю обратной задачи рассеяния $(\mathrm{O} 3 \mathrm{P})$. В этих двух случаях операторы в гильбертовом пространстве однозначно определяются по данным дифференциальным и дискретным разностным операторам. Действительно, при определенных ограничениях вешественности и асимптотических требованиях вида (1.7) соответствуюшие операторы Якоби явно ограничены и самосопряжены на $l^{2}(\mathbb{Z})$, а любой оператор Шредингера $-\partial_{x}^{2}+V(x)$ является, очевидно, самосопряженным на естественной области (пространстве Соболева) оператора $\partial_{x}^{2}$, коль скоро $V(x)$ ограничен и вещественнозначен. Таким образом, безотражательные собственные функции приводят к преобразованиям собственных функций, дающим явную реализацию спектральной теоремы для однозначно определенных самосопряженных операторов соответственно на $l^{2}(\mathbb{Z})$ и $L^{2}(\mathbb{R}, d x)$.

Как видно из нашего обсуждения случая свободного оператора (1.1), ситуация с $\mathrm{A} \Delta \mathrm{O}$ носит иной характер. Возврашаясь к сформулированным выше вопросам, отметим прежде всего, что первые два ранее не изучались. Из наших результатов вытекает сушествование широкого класса $\mathrm{A} \Delta \mathrm{O}$, для которых имеется положительный ответ на второй вопрос. Но нам представляется очень маловероятно, чтобы это давало наиболее обший класс $\mathrm{A} \Delta \mathrm{O}$, допускающих безотражательные собственные функции.

Подобным же образом мы частично ответим на третий вопрос, показав, что при весьма ограничительных дополнительных условиях наши преобразования безотражательных собственных функций оператора $\mathcal{F}$ являются изометриями и поэтому могут использоваться для того, чтобы связать с $A$ самосопряженный оператор $\hat{A}$ на $L^{2}(\mathbb{R}, d x)$, сопрягая оператор умножения $M$, действуюший на $L^{2}(\mathbb{R}, d p)$. Точнее говоря, этой процедуры достаточно, коль скоро оператор $\mathcal{F}$ отображает на $L^{2}(\mathbb{R}, d x)$. Это имеет место для бесконечномерного пространства потенциалов $\left(V_{a}, V_{b}\right)$, что снова резко отличается от случая операторов Шредингера и Якоби, для которых нетривиальные безотражательные потенциалы всегда имеют связанные состояния.

В случае, когда оператор $\mathcal{F}$ не является отображением на все пространство $L^{2}(\mathbb{R}, d p)$, можно показать, что ортогональное дополнение к $\mathcal{F}\left(L^{2}(\mathbb{R}, d p)\right)$ порождается конечным числом попарно ортогональных собственных функций $A$ с вешественными собственными значениями; поэтому можно определить $\hat{A}$ равным $A$ на этом подпространстве связанных состояний. После введения подходящего изменения масштаба в $\mathrm{A} \Delta \mathrm{O}$ можно показать, что операторы такого типа сходятся к классу безотражательных операторов Шредингера при стремяшемся к нулю шаге. Мы упоминаем последний результат (который подробно рассматривается в разделе 3 работы [5]) для того, чтобы с самого начала было ясно, что класс самосопряженных безотражательных $\mathrm{A} \Delta \mathrm{O}$, который мы строим, является весьма большим.

Тем не менее вполне может оказаться, что данный $\mathrm{A} \Delta \mathrm{O} A$ в этом классе дает совсем различные самосопряженные безотражательные операторы на $L^{2}(\mathbb{R}, d x)$. (Как объяснялось выше, для специальных $\mathrm{A} \Delta \mathrm{O} A_{0}$, задаваемых формулой (1.1), действительно существует бесконечномерное пространство таких операторов.) Наши результаты долж- 
ны быть полезны при изучении прямой задачи, но случай $b(p) \neq 0$ еще не исследовался. Как нам кажется, наши результаты могут открывать начало (вероятно, весьма богатой) теории операторов в гильбертовых пространствах для $\mathrm{A} \Delta \mathrm{O}$ указанного простого вида. Из конкретных случаев, которые мы смогли рассмотреть, ясно видно, что основной задачей в данной области является выделение изометрических преобразований собственных функций.

Данная работа организована следуюшим образом. В разделе 2 мы даем набросок нашей конструкции для широкого класса $\mathrm{A} \Delta \mathrm{O}$ вида (1.6), допускающих безотражательные собственные функции, которые также строятся явно. Мы представляем все детали в простейшем случае $(N=1)$. Основная идея состоит в том, чтобы следовать схеме ОЗР для безотражательных операторов Шредингера и Якоби [7]-[11].

В разделе 3 объясняется связь безотражательных А $\Delta \mathrm{O}$ с новым солитонным эволюционным уравнением, которое можно рассматривать как непрерывный нелокальный вариант бесконечных уравнений решетки Тоды. Мы получаем вешественнозначные $N$ солитонные решения посредством подходяших ограничений на спектральные данные.

При наложенных ограничениях на спектральные данные мы рассматриваем функционально-аналитические свойства в разделе 4 . Здесь подробно исследуется специальный случай $N=1$ и дается оценка того, как предыдущие работы [1]-[3] укладываются в ньнешнюю более общую схему.

\section{2. ПОСТРОЕНИЕ БЕЗОТРАЖАТЕЛЬНЫХ А $\Delta O$}

Будем исходить из “спектральных данных"

$$
(r, \mu)=\left(r_{1}, \ldots, r_{N}, \mu_{1}(x), \ldots, \mu_{N}(x)\right), \quad N \in \mathbb{N}^{*}
$$

на которые наложены следуюшие ограничения. Комплексные числа $r_{1}, \ldots, r_{N}$ удовлетворяют условиям

$$
\operatorname{Im} r_{n} \in(-\pi, 0) \cup(0, \pi), \quad n=1, \ldots, N,
$$

и

$$
e^{r_{m}} \neq e^{ \pm r_{n}}, \quad 1 \leqslant m<n \leqslant N .
$$

Функции $\mu_{1}(x), \ldots, \mu_{N}(x)$ ("нормировочные коэффициенты") могут быть мероморфными, причем

$$
\mu_{n}(x+i)=\mu_{n}(x), \quad \lim _{|\operatorname{Re} x| \rightarrow \infty} \mu_{n}(x)=c_{n}, \quad c_{n} \in \mathbb{C}^{*}, \quad n=1, \ldots, N
$$

(Аналог операторов Шредингера и Якоби возникает в специальном случае $\mu_{n}(x)=c_{n}$, $n=1, \ldots, N$, который, разумеется, включен в наше рассмотрение.)

Из условия на $r$ следует, что матрица Коши

$$
C(r)_{m n} \equiv \frac{1}{e^{r_{m}}-e^{-r_{n}}}, \quad m, n=1, \ldots, N
$$


корректно определена и регулярна, как в случае операторов Шредингера и Якоби. Определим далее диагональную матрицу

$$
D(r, \mu ; x) \equiv \operatorname{diag}\left(d_{1}(x), \ldots, d_{N}(x)\right),
$$

где использовано обозначение

$$
d_{n}(x)=d\left(r_{n}, \mu_{n} ; x\right), \quad n=1, \ldots, N,
$$

при

$$
d(\rho, \nu ; x) \equiv \begin{cases}\nu(x) e^{-2 i \rho x}, & \operatorname{Im} \rho \in(0, \pi), \\ \nu(x) e^{-2 i(\rho+i \pi) x}, & \operatorname{Im} \rho \in(-\pi, 0) .\end{cases}
$$

Все остальные величины определяются теперь из решения $R(r, \mu ; x)$ системы

$$
(D(r, \mu ; x)+C(r)) R=\zeta, \quad \zeta \equiv(1, \ldots, 1)^{\mathrm{t}} .
$$

В терминах вспомогательных функций

$$
\begin{aligned}
\lambda(r, \mu ; x) & \equiv 1+\sum_{n=1}^{N} e^{r_{n}} R_{n}(r, \mu ; x), \\
\Sigma(r, \mu ; x) & \equiv \sum_{n=1}^{N} R_{n}(r, \mu ; x)
\end{aligned}
$$

определяюшие $\mathrm{A} \Delta \mathrm{O} \quad A$ посредством (1.6) потенциалы $V_{a}$ и $V_{b}$ с заданной асимптотикой (1.7) имеют вид

$$
\begin{aligned}
V_{a}(r, \mu ; x) & \equiv \frac{\lambda(r, \mu ; x)}{\lambda(r, \mu ; x+i)}, \\
V_{b}(r, \mu ; x) & \equiv \Sigma(r, \mu ; x-i)-\Sigma(r, \mu ; x) .
\end{aligned}
$$

Более того, волновая функция, удовлетворяюшая условию (1.8), имеет вид

$$
\mathcal{W}(r, \mu ; x, p) \equiv e^{i x p}\left(1-\sum_{n=1}^{N} \frac{R_{n}(r, \mu ; x)}{e^{p}-e^{-r_{n}}}\right) .
$$

Она имеет асимптотику $(1.9)$ при $b(p)=0$ и

$$
a(p)=\prod_{n=1}^{N} \frac{e^{p}-e^{r_{n}}}{e^{p}-e^{-r_{n}}} .
$$

В дальнейшем нам также понадобится асимптотика

$$
\lambda(x) \sim \begin{cases}1, & \operatorname{Re} x \rightarrow \infty, \\ \exp \left(2 \sum_{n=1}^{N} r_{n}\right), & \operatorname{Re} x \rightarrow-\infty .\end{cases}
$$


Разумеется, ни утверждение об асимптотических свойствах, ни утверждение о собственных значениях не являются очевидными. Они следуют из подробного анализа $(N \times N)$-линейной системы (2.6) (см. раздел 2 работы [4]). Здесь же мы разберем только случай $N=1$. В этом специальном случае соответствуюшая асимптотика получается немедленно, но доказательство свойств собственных значений уже не является тривиальным. Более того, это доказательство весьма поучительно, поскольку демонстрирует основной момент доказательства для произвольного $N$ и указывает пути дальнейших обобщений.

Итак, при $N=1$ необходимо рассмотреть два случая: $\operatorname{Im} r>0$ и $\operatorname{Im} r<0$. В первом случае получаем

$$
\begin{gathered}
C=\frac{1}{2 \operatorname{sh} r}, \quad d(x)=\mu(x) e^{-2 i r x}, \quad \operatorname{Im} r \in(0, \pi), \\
\mu(x) \in \mathcal{M}, \quad \mu(x+i)=\mu(x), \quad \lim _{|\operatorname{Re} x| \rightarrow \infty} \mu(x)=c \in \mathbb{C}^{*} .
\end{gathered}
$$

Отсюда имеем

$$
R(x)=\left[\mu(x) e^{-2 i r x}+\frac{1}{2 \operatorname{sh} r}\right]^{-1} \sim \begin{cases}0, & \operatorname{Re} x \rightarrow \infty \\ 2 \operatorname{sh} r, & \operatorname{Re} x \rightarrow-\infty\end{cases}
$$

Теперь из (2.7) и (2.8) следует, что

$$
\begin{array}{lll}
V_{a}(x)=\frac{1+e^{r} R(x)}{1+e^{r} R(x+i)} \sim 1, & & |\operatorname{Re} x| \rightarrow \infty, \\
V_{b}(x)=R(x-i)-R(x) \sim 0, & & |\operatorname{Re} x| \rightarrow \infty .
\end{array}
$$

Аналогично из (2.9) получаем

$$
\mathcal{W}(x, p)=e^{i x p}\left(1-\frac{R(x)}{e^{p}-e^{-r}}\right) \sim \begin{cases}e^{i x p}, & \operatorname{Re} x \rightarrow \infty, \\ \frac{e^{p}-e^{r}}{e^{p}-e^{-r}} e^{i x p}, & \operatorname{Re} x \rightarrow-\infty,\end{cases}
$$

как и утверждалось.

Во втором случае $\operatorname{Im} r \in(-\pi, 0)$, и отличия от первого случая возникают лишь в формулах для $d(x)$ и $R(x)$ :

$$
R(x)=\left[\mu(x) e^{-2 i r x+2 \pi x}+\frac{1}{2 \operatorname{sh} r}\right]^{-1}, \quad \operatorname{Im} r \in(-\pi, 0) .
$$

Докажем теперь свойства собственных функций оператора $A$, рассматривая оба случая одновременно. Нам надо показать, что функция $\mathcal{W}(x, p)$ удовлетворяет аналитическому разностному уравнению второго порядка

$$
F(x-i)+V_{a}(x) F(x+i)+\left[V_{b}(x)-e^{p}-e^{-p}\right] F(x)=0 .
$$

Для этого достаточно доказать, что вспомогательная волновая функция

$$
\mathcal{A}(x, p) \equiv\left(e^{p}-e^{-r}\right) \mathcal{W}(x, p)=e^{i x p}\left(e^{p}-e^{-r}-R(x)\right)
$$


удовлетворяет уравнению (2.14). Для этого подставим $F(x)=\mathcal{A}(x, p)$ в левую часть (2.14) и получим функцию $\mathcal{D}(x, p)$ вида

$$
\mathcal{D}(x, p)=e^{i x p}\left(e^{p} c_{1}(x)+c_{0}(x)+e^{-p} c_{-1}(x)\right) .
$$

Очевидно, что для обращения этой функции в нуль необходимо и достаточно, чтобы коэффициенты $c_{1}, c_{0}$ и $c_{-1}$ обрашались в нуль. Далее, легко проверить эквивалентности

$$
\begin{gathered}
c_{1}(x)=0 \quad \Leftrightarrow \quad V_{b}(x)=R(x-i)-R(x), \\
c_{-1}(x)=0 \quad \Leftrightarrow \quad V_{a}(x)=\frac{1+e^{r} R(x)}{1+e^{r} R(x+i)} .
\end{gathered}
$$

Поскольку мы определили потенциалы $V_{a}$ и $V_{b}$ так, что равенства в правых частях выполнены, то можно заключить, что коэффициенты $c_{1}$ и $c_{-1}$ обращаются в нуль.

Нетривиальное утверждение состоит в том, что коэффициент $c_{0}(x)$ также обращается в нуль. Даже в данном весьма простом случае непосредственная проверка этого связана с объемными вычислениями. Но прямой проверки можно избежать, если обратиться к аргументу единственности, который обобщается на случай произвольного $N$. А именно, сначала заметим, что поскольку $R(x)$ удовлетворяет системе вида $(2.6)$ при $N=1$, то из $(2.15)$ и (2.5) следует, что

$$
\begin{gathered}
\mathcal{A}(x, r)=e^{i r x}\left(e^{r}-e^{-r}\right)(1-C R(x))= \\
=e^{i r x}\left(e^{r}-e^{-r}\right) d(x) R(x)=\alpha(x) \mathcal{A}(x,-r), \\
\alpha(x+i)=\alpha(x) .
\end{gathered}
$$

Основным моментом здесь является тот факт, что функция

$$
G(x, p)=e^{i x p}\left(e^{p}+c(x)\right)
$$

удовлетворяющая соотношению

$$
G(x, r)=\alpha(x) G(x,-r)
$$

для данной $i$-периодической функции $\alpha(x)$, единственна. Действительно, подстановка $(2.17)$ в (2.18) показывает, что $c(x)$ определяется единственным образом. Чтобы использовать это обстоятельство, вспомним, что, как было показано вьше, как $\mathcal{A}(x, p)$, так и $\mathcal{A}(x, p)-\mathcal{D}(x, p)$ имеют вид $(2.17)$. Кроме того, функция $\mathcal{A}(x, p)$ удовлетворяет соотношению (2.18) ввиду (2.16). Далее, функция $\mathcal{D}(x, p)$ возникает из подстановки $F(x) \rightarrow \mathcal{A}(x, p)$ в левую часть уравнения $(2.14)$, и поскольку $\alpha(x)$ является $i$ периодической функцией, то и функция $\mathcal{D}(x, p)$ также удовлетворяет $(2.18)$. Но тогда как $\mathcal{A}(x, p)$, так и $\mathcal{A}(x, p)-\mathcal{D}(x, p)$ удовлетворяют $(2.18)$, а потому $\mathcal{A}(x, p)=\mathcal{A}(x, p)-\mathcal{D}(x, p)$ в силу единственности. Отсюда $\mathcal{D}(x, p)=0$, что и завершает доказательство.

Подчеркнем, что предположение о том, что $\mu(x) \rightarrow c \in \mathbb{C}^{*}$ при $|\operatorname{Re} x| \rightarrow \infty$, не используется в этом доказательстве (а $i$-периодичность $\mu(x)$, наоборот, используется). В результате можно расширить указанный вьше класс безотражательных $\mathrm{A} \Delta \mathrm{O}$ при $N=1$ 
посредством ослабления требований на $\mu(x)$. Например, легко проверить, что, выбирая $\mu(x) \equiv c_{1}+c_{2} \operatorname{th} \pi\left(x-x_{0}\right)$, приходим к тем же заключениям. Выбирая же вместо этого $\mu(x) \equiv c_{1}+c_{2} \operatorname{ch} 4 \pi\left(x-x_{0}\right)$, получаем, что $R(x) \rightarrow 0$ при $|\operatorname{Re} x| \rightarrow \infty$, а потому условия (1.7)-(1.9) справедливы при $a(p)=1$ и $b(p)=0$.

Последний пример показывает, что взаимодействующий $\mathrm{A} \Delta \mathrm{O} A$, задаваемый формулой (1.6) (т.е. с потенциалами $\left(V_{a}, V_{b}\right) \neq(1,0)$, удовлетворяюшими соотношениям (1.7)), может допускать собственные функции $\mathcal{W}(x, p)$ с тривиальным рассеянием.

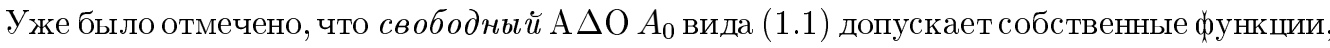
которые приводят к нетривиальному рассеянию, и унитарное преобразование собственных функций. Любая обшая теория, основанная на соотношениях (1.6) и (1.7), должна учитывать это явление, не имеюшее аналогов для операторов Шредингера и Якоби.

Нашей целью в работах [4]-[6] было выделение специального класса безотражательных $\mathrm{A} \Delta \mathrm{O}$, свойства которых можно описать весьма детально. Ограничения (2.1)-(2.4) на спектральные данные определяют этот специальный класс, который уже является достаточно большим и который можно систематически изучать с алгебраической точки зрения [4]. Для дальнейших целей отметим два обших аспекта, относяшихся к объектам в этом классе.

Во-первых, наличие и положение возникаюших в конце концов полюсов по $x$ функции $\mathcal{W}(x, p)$ определяется наличием и положением нулей множителей $\mu_{1}(x), \ldots, \mu_{N}(x)$ и $\tau$ функции

$$
\tau(x) \equiv\left|\mathbf{1}_{N}+C D(x)^{-1}\right| .
$$

Действительно, это следует из соотношения (2.9), если разрешить систему (2.6) в соответствии с правилом Крамера.

Второй аспект относится к условиям, обеспечивающим формальную самосопряженность $\mathrm{A} \Delta \mathrm{O} A$ вида (1.6) на $L^{2}(\mathbb{R}, d x)$ безотносительно областей определения, сингулярностей и т. п. Очевидно, требуется, чтобы потенциал $V_{b}(x)$ был вешественнозначным при вешественных $x$ и, кроме того, чтобы

$$
\left[V_{a}(x) e^{i \partial_{x}}\right]^{*}=e^{i \partial_{x}} \overline{V_{a}(x)}=\overline{V_{a}(x-i)} e^{i \partial_{x}}, \quad x \in \mathbb{R} .
$$

В обозначениях

$$
f^{*}(x) \equiv \overline{f(\bar{x})}, \quad x \in \mathbb{C}, \quad f \in \mathcal{M},
$$

формальная самосопряженность обеспечивается условиями

$$
V_{b}^{*}(x)=V_{b}(x), \quad V_{a}^{*}(x)=V_{a}(x-i) .
$$

Далее можно показать, что это эквивалентно условиям

$$
\begin{aligned}
\operatorname{Re}\left(r_{n}\right) & =0, \quad n=1, \ldots, N, \\
\operatorname{Re}\left(e^{-r_{n}} \mu_{n}(x)\right) & =0, \quad n=1, \ldots, N, \quad x \in \mathbb{R}
\end{aligned}
$$

(см. приложение $\mathrm{D}$ в работе [4]). При $N=1$ эти утверждения легко проверить непосредственно исходя из приведенных выше явных формул. 


\section{3. СВЯЗАННОЕ НЕЛОКАЛЬНОЕ СОЛИТОННОЕ УРАВНЕНИЕ ТИПА ТОДЫ}

Чтобы установить связь обсуждаемых выше объектов с солитонными эволюционными уравнениями, надо ввести подходящую зависимость от времени в нормировочные коэффициенты $\mu(x)$. Точнее говоря, такова процедура, приводящая к солитонным решениям решетчатых уравнений Кортевега-де Фриза (КдФ) и Тоды для случая безотражательных операторов Шредингера и Якоби. Рассматривая ситуацию с этими дискретными разностными операторами как указание на правильный способ действий, определим временну́ю эволюцию как

$$
\mu_{n}(x) \rightarrow \mu_{n}(x) e^{2 i t \operatorname{sh} r_{n}}, \quad n=1, \ldots, N
$$

После этой подстановки все величины $R, V_{a}, V_{b}, \mathcal{W}$ и $\tau$ зависят от $t$. Для упрошения обозначений мы будем, как правило, опускать эту временну́ю зависимость. Снова используя свойства, вытекающие только из системы (2.6) (теперь зависящей от времени), можно получить уравнения

$$
\begin{gathered}
\dot{V}_{a}(x)=i V_{a}(x)\left[V_{b}(x+i)-V_{b}(x)\right], \quad \dot{V}_{b}(x)=i\left[V_{a}(x)-V_{a}(x-i)\right], \\
\dot{\mathcal{W}}(x, p)=(B \mathcal{W})(x, p)+i e^{p} \mathcal{W}(x, p), \quad B \equiv-i\left(e^{-i \partial_{x}}+V_{b}(x)\right)
\end{gathered}
$$

и

$$
\ddot{\tau}(x) \tau(x)-\dot{\tau}(x)^{2}=\tau(x)^{2}-\tau(x+i) \tau(x-i) .
$$

(См. раздел 2 работы [5]; эти уравнения легко проверить непосредственно при $N=1$.)

Очевидно, уравнение (3.1) является билинейной формой уравнения Хироты. Переписывая это уравнение как

$$
\partial_{t}^{2} \ln \tau(x)=1-\frac{\tau(x+i) \tau(x-i)}{\tau(x)^{2}}
$$

и вводя функцию

$$
\Psi(x) \equiv i \ln \left(\frac{\tau(x-i)}{\tau(x)}\right),
$$

легко найти, что функция $\Psi$ удовлетворяет нелокальному уравнению типа Тоды

$$
\ddot{\Psi}(x)=i e^{i[\Psi(x+i)-\Psi(x)]}-i e^{i[\Psi(x)-\Psi(x-i)]} .
$$

(Подобные нелокальные эволюционные уравнения изучались и ранее; см., например, обзор Сантини [12] и приведенную там литературу.)

Можно показать, что требование вещественнозначности функции $\Psi(x, t)$ при вещественных $x$ и $t$ удовлетворяется, если $\mathrm{A} \Delta \mathrm{O} A(r, \mu(x, t))$ является формально самосопряженным, т.е. если выполнены условия (2.20) и (2.21). (Дело в том, что эти условия обеспечивают вещественнозначность $\tau(x-i / 2)$ при вещественных $x$.) 
Рассмотрим теперь решение для $N=1$ при $\operatorname{Im} r \in(0, \pi)$ :

$$
\Psi(x, t)=i \ln \left(\frac{1+(2 \operatorname{sh} r)^{-1}(\mu(x))^{-1} e^{2 i r(x-i)-2 i t \operatorname{sh} r}}{1+(2 \operatorname{sh} r)^{-1}(\mu(x))^{-1} e^{2 i r x-2 i t} \operatorname{sh} r}\right) .
$$

Если $\mu(x)$ не является константой, эта функция не имеет вида бегущей волны $f(x-v t)$. Однако полагая

$$
r=i \alpha, \quad \alpha \in(0, \pi), \quad \mu(x)=\frac{e^{i \alpha}}{2 \operatorname{sh}(i \alpha)} e^{-2 \alpha x_{0}}, \quad x_{0} \in \mathbb{R}
$$

получаем движущийся направо солитон типа кинка

$$
\Psi(x, t)=i \ln \left(\frac{1+e^{i \alpha} e^{-2 \alpha\left[\left(x-x_{0}\right)-v(\alpha) t\right]}}{1+e^{-i \alpha} e^{-2 \alpha\left[\left(x-x_{0}\right)-v(\alpha) t\right]}}\right), \quad v(\alpha) \equiv \frac{\sin \alpha}{\alpha} .
$$

Выбирая

$$
r=i \alpha-i \pi, \quad \alpha \in(0, \pi), \quad \mu(x)=-\frac{e^{i \alpha}}{2 \operatorname{sh}(i \alpha)} e^{-2 \alpha x_{0}}, \quad x_{0} \in \mathbb{R},
$$

подобным же образом получаем соотношение (3.3) с заменой $t \rightarrow-t$ в правой части, т.е. движущийся налево солитон.

Обрашаясь к случаю произвольного $N$, выберем спектральные данные вида (3.2) для $N_{+} \in\{0,1, \ldots, N\}$ чисел, выбранных из $r_{1}, \ldots, r_{N}$, и вида (3.4) для $N_{-} \equiv N-N_{+}$ оставшихся (этот выбор гарантирует, что выполнены условия (2.20) и (2.21) и функция $\Psi(x, t)$ является поэтому вещественнозначной при вешественных $x, t)$. Тогда функцию $\Psi(x, t)$ можно рассматривать как $N$-солитонное решение: его асимптотика на больших временах содержит $N_{+}$движушихся направо и $N_{-}$движушихся налево 1-кинковых решений, подробно рассмотренных выше.

Справедливость последнего утверждения не является очевидной, но его достаточно просто доказать в слабой форме (см. предложение 6.1 в работе [5]). Более подробньй анализ (включаюший изучение траекторий солитонов в пространстве-времени) опирается на довольно замысловатую репараметризацию $2 N$ вешественных чисел $\alpha_{1}, \ldots, \alpha_{N}, x_{0,1}, \ldots, x_{0, N}$, в терминах которых определяется функция $\Psi(x, t)$. Эта репараметризация играет основную роль и при изучении вопросов самосопряженности. Мы продолжим ее описание в обшем виде. (Подробности можно найти в разделе 5 работы [5].)

Для этого вспомним, что хорошо известные $N$-частичные системы КалоджероМозера допускают обобщение на релятивистский случай [13]. Как и в нерелятивистском случае, имеется решение, описывающее $N_{+}$частиц и $N_{-}$античастиц, в том смысле, что частица и античастица притягивают друг друга, тогда как две частицы или две античастищы отталкиваются. В работе [14] мы подробно рассмотрели это решение, а также частище-подобные решения уравнений КдФ, синус-Гордон и модифицированного КдФ. Действительно, последние решения солитонного типа можно также параметризовать релятивистскими $N$-частичными системами; такое соответствие солитон-частица можно очень эффективно использовать (см. раздел 7 в работе [14]). 
Репараметризация, требуемая для указанных выше нелокальных солитонов Тоды, означает, что $\tau$-функция (2.19) связана с матрицей Лакса $L$ систем частиц следуюшим образом:

$$
\tau\left(x-\frac{i}{2}, t\right)=\left|\mathbf{1}_{N}+L(x, t)\right|, \quad x, t \in \mathbb{R} .
$$

Здесь $L(x, t)$ обозначает матрицу Лакса, вычисленную в зависящей от $(x, t)$ точке соответствуюшего $2 N$-мерного фазового пространства. Если только числа $N_{+}$или $N_{-}$не обрашаются в нуль, это соотношение является новым. Действительно, в предыдущих случаях солитонные $\tau$-функции были связаны с дуальнымми матрицами Лакса систем частиц (см. также [15]). В нашем случае, к сожалению, аналитические следствия соответствия солитон-частица (выражаемые формулой (3.5)) представляются не такими эффективными, как ранее. В частности, даже когда числа $N_{+}$или $N_{-}$обрашаются в нуль, не удается получить равномерные ограничения на асимптотическое поведение на больших временах. Более того, четкие траектории солитонов в пространстве-времени сушествуют только при достаточно больших временах (за исключением случаев, когда числа $N_{+}$или $N_{-}$обрашаются в нуль).

\section{4. ИЗОМЕТРИЯ И ВОПРОСЫ САМОСОПРЯЖЕННОСТИ}

Сохраняя сделанный ранее выбор спектральных данных, приводяший к $N$-солитонным решениям, изучим теперь свойства гильбертова пространства (доказательства делаемых ниже утверждений см. в работе [6]). Основное свойство, требуемое для установления ортогональности и полноты, заключается в отсутствии полюсов функции $\mathcal{W}(x, p)$ в полосе $\operatorname{Im} x \in[-1,0]$. Объясним сначала, как можно использовать соотношение $(3.5)$ при $t=0$ для исследования этого вопроса о полюсах.

Для этого заметим, что отсутствие полюсов в критической полосе гарантировано отсутствием нулей функции $\tau(x)$ в той же полосе. В самом деле, это следует из рассуждения, содержащего формулу (2.19) (напомним, что в данном разделе $\mu_{1}(x), \ldots, \mu_{N}(x)$ - константы). Согласно (3.5) таких нулей нет, если спектр $L(x)$ не содержит числа -1 при $\operatorname{Im} x \in[-1 / 2,1 / 2]$.

Здесь можно привлечь спектральный анализ матриц Лакса [14]. А именно, можно сделать вывод об отсутствии полюсов при условии, что

$$
r_{n} \in i(-\pi,-\pi / 2) \cup i(0, \pi / 2), \quad n=1, \ldots, N .
$$

Это условие можно ослабить до

$$
r_{n} \in i(0, \pi), \quad n=1, \ldots, N
$$

при $N=N_{+}$и до

$$
r_{n} \in i(-\pi, 0), \quad n=1, \ldots, N
$$

при $N=N_{-}$. Аналогичным образом в качестве спектральных данных в двух последних случаях можно выбрать произвольные точки фазового пространства. Представляется 
вероятным, что условие (4.1) можно значительно ослабить, но при $N_{+} N_{-}>0$ сушествуют точки фазового пространства, приводящие к нулям функции $\tau$ в критической полосе.

Ниже мы будем считать, что спектральные данные таковы, что функция $\tau(x)$ не имеет нулей в указанной полосе. Рассмотрим подробнее некоторые наиболее характерные свойства гильбертова пространства. Во-первых, собственные функции $\mathcal{W}\left(x, r_{n}\right)$ оператора $A$ при $r_{n} \in i(0, \pi)$ лежат в $L^{2}(\mathbb{R}, d x)$ и являются попарно ортогональными. Вовторых, преобразование собственных функций

$$
\mathcal{F}: \quad L^{2}(\mathbb{R}, d p) \rightarrow L^{2}(\mathbb{R}, d x), \quad f(p) \mapsto(2 \pi)^{-\frac{1}{2}} \int_{-\infty}^{\infty} d p \mathcal{W}(x, p) f(p)
$$

является изометрическим. В-третьих, ортогональное дополнение к $\mathcal{F}\left(L^{2}(\mathbb{R}, d p)\right)$ есть линейная оболочка связанных состояний $\mathcal{W}\left(x, r_{n}\right)$ при $r_{n} \in i(0, \pi)$ (в частности, при $N_{+}=0$ нет связанных состояний, а преобразование $\mathcal{F}$ является унитарным).

Эти свойства позволяют связать самосопряженный оператор $\hat{A}$ на $L^{2}(\mathbb{R}, d x)$ с $\mathrm{A} \Delta \mathrm{O}$ $A$ на $\mathcal{M}$ следуюшим образом. Определим $\hat{A}$ как умножение на $2 \operatorname{ch} r_{n}$ на $N_{+}$связанных состояниях $\mathcal{W}\left(x, r_{n}\right)$ при $r_{n} \in i(0, \pi)$. На ортогональном дополнении $\mathcal{F}\left(L^{2}(\mathbb{R}, d p)\right)$ к подпространству связанных состояний определим оператор $\hat{A}$ как самосопряженный оператору умножения $M$ на $L^{2}(\mathbb{R}, d p)$ с областью определения $\mathcal{D}(M)$ :

$$
\hat{A} \mathcal{F} f \equiv \mathcal{F} M f \quad \forall f \in \mathcal{D}(M) .
$$

Теперь легко проверить, что при $f(p) \in C_{0}^{\infty}(\mathbb{R})$ функция $(\mathcal{F} f)(x)$ принадлежит $\mathcal{M}$ и что действие $\hat{A}$ на $\mathcal{F} f$ совпадает с действием $A$ точно так же, как и действие $\hat{A}$ на связанных состояниях.

Рассмотрим подробно случай $N=1$. Этот случай поучителен, в частности, потому, что различную природу выбора $N=N_{+}$и $N=N_{-}$легко проиллюстрировать при $N=1$. Выбирая $N=N_{+}$(см. (3.2)), можно положить $x_{0}=0$, поскольку $x_{0}$ является просто параметром сдвига. Тогда из (2.9) и (2.11) находим, что

$$
\mathcal{W}_{+}(x, p)=e^{i x p} \frac{e^{i \alpha} \operatorname{sh}\left(\alpha x+\frac{p}{2}\right)-\operatorname{sh}\left(\alpha x-\frac{p}{2}\right)}{2 \operatorname{ch}\left(\alpha x+\frac{i \alpha}{2}\right) \operatorname{sh}\left(\frac{p}{2}+\frac{i \alpha}{2}\right)}, \quad N=N_{+}=1 .
$$

Подобным же образом выбор $N=N_{-}$(3.4) с учетом (2.9) и (2.13) приводит к

$$
\mathcal{W}_{-}(x, p)=e^{i x p} \frac{e^{i \alpha} \operatorname{ch}\left(\alpha x+\frac{p}{2}\right)+\operatorname{ch}\left(\alpha x-\frac{p}{2}\right)}{2 \operatorname{ch}\left(\alpha x+\frac{i \alpha}{2}\right) \operatorname{ch}\left(\frac{p}{2}+\frac{i \alpha}{2}\right)}, \quad N=N_{-}=1 .
$$

Из этих явных формул получаем

$$
\mathcal{W}_{ \pm}(x, p) \sim e^{i x p}, \quad \operatorname{Re} x \rightarrow \infty
$$

и

$$
\mathcal{W}_{+}(x, p) \sim e^{i \alpha} \frac{\operatorname{sh}\left(\frac{p}{2}-\frac{i \alpha}{2}\right)}{\operatorname{sh}\left(\frac{p}{2}+\frac{i \alpha}{2}\right)} e^{i x p}, \quad \mathcal{W}_{-}(x, p) \sim e^{i \alpha} \frac{\operatorname{ch}\left(\frac{p}{2}-\frac{i \alpha}{2}\right)}{\operatorname{ch}\left(\frac{p}{2}+\frac{i \alpha}{2}\right)} e^{i x p}, \quad \operatorname{Re} x \rightarrow-\infty
$$


в соответствии с (2.12). Для квадратичной интегрируемости при $x=\infty$ требуется $\operatorname{Im} p>0$. Это ограничение на $p$ может быть совместно с квадратичной интегрируемостью при $x=-\infty$, только если $p=i \alpha+2 k i \pi, k \in \mathbb{N}$, для $\mathcal{W}_{+}$и $p=i \alpha+(2 k+1) i \pi$, $k \in \mathbb{N}$, для $\mathcal{W}_{-}$. Тогда получаем

$$
\begin{aligned}
\mathcal{W}_{+}(x, i \alpha+2 k i \pi) & =\frac{e^{-2 k \pi x}}{e^{\alpha x}+e^{-i \alpha-\alpha x}}, \quad \alpha \in(0, \pi), \quad k \in \mathbb{Z}, \\
\mathcal{W}_{-}(x, i \alpha+(2 k+1) i \pi) & =\frac{e^{-(2 k+1) \pi x}}{e^{\alpha x}+e^{-i \alpha-\alpha x}}, \quad \alpha \in(0, \pi), \quad k \in \mathbb{Z} .
\end{aligned}
$$

Поэтому следует выбрать $k=0$, чтобы соотношение (4.4) приводило к функции из $L^{2}(\mathbb{R}, d x)$, тогда как формула (4.5) не дает квадратично-интегрируемой функций для любого $k \in \mathbb{Z}$.

Соотношение (4.3) можно использовать для иллюстрации еше одного обстоятельства. При

$$
\mathcal{W}_{-}\left(\frac{\pi x}{\alpha}, \frac{\alpha p}{\pi}\right)=\mu(x, p) e^{i x p}
$$

функция $\mu(x, p)$ является, очевидно, $i$-периодической и имеет вид

$$
\mu(x, p) \sim \begin{cases}1, & \operatorname{Re} x \rightarrow \infty, \\ \frac{e^{\frac{\alpha p}{\pi}}+e^{i \alpha}}{e^{\frac{\alpha p}{\pi}}+e^{-i \alpha}}, & \operatorname{Re} x \rightarrow-\infty,\end{cases}
$$

т.е. удовлетворяет условиям (1.4). Поскольку функция $(2 \pi)^{-1 / 2} \mathcal{W}_{-}(x, p)$ является ядром унитарного оператора, это свойство выполнено и для масштабированного ядра $(2 \pi)^{-1 / 2} \mu(x, p) e^{i x p}$. В результате в гильбертовом пространстве получаем пример взаимодействуюшего самосопряженного оператора $\hat{A}_{\mu}$ вида (1.5), связанного со свободным А $\Delta \mathrm{O} A_{0}$ вида (1.1).

Кратко остановимся теперь на вопросе о том, как соглашения и результаты работы [3] связаны с приведенными выше. Тройку параметров $(\hbar, \nu, \beta)$, использованных в работе [3], следует положить равными $(1, \alpha, 1 / 2)$. Заметим, что приведенные выше функции $\mathcal{W}_{ \pm}(x, p)$ можно также рассматривать как обшие собственные функции взаимодействуюших $\mathrm{A} \Delta \mathrm{O} A_{ \pm}$и свободного $\mathrm{A} \Delta \mathrm{O}$

$$
A_{\mathrm{f}} \equiv e^{i \pi \alpha^{-1} \partial_{x}}+e^{-i \pi \alpha^{-1} \partial_{x}}
$$

с собственными значениями $2 \operatorname{ch} p$ и $2 \operatorname{ch}(\pi p / \alpha)$. После унитарного преобразования подобия собственные функции из работы [3] сводятся к волновым функциям $\mathcal{W}(x, p)$ описанного в разделе 2 вида с одним специальным свойством, заключаюшимся в том, что все они суть обшие собственные функции $A$ и $A_{\mathrm{f}}$ с указанными собственными значениями.

Целесообразно подробно рассмотреть случай $N=1$, прежде чем описьвать ситуацию произвольного $N$. При вешественных $x$ и $p$ и при $N=1$ функции $F_{a}(x, p)$ и $F_{e}(x, p)$ 
из разделов 4 и 5 работы [3] имеют вид

$$
\begin{aligned}
& F_{a}(x, p)=e^{-\frac{i \alpha}{2}}\left(\frac{\operatorname{ch}\left(\alpha x+\frac{i \alpha}{2}\right)}{\operatorname{ch}\left(\alpha x-\frac{i \alpha}{2}\right)}\right)^{\frac{1}{2}}\left(\frac{\operatorname{sh}\left(\frac{p}{2}+\frac{i \alpha}{2}\right)}{\operatorname{sh}\left(\frac{p}{2}-\frac{i \alpha}{2}\right)}\right)^{\frac{1}{2}} \mathcal{W}_{+}(x, p), \\
& F_{e}(x, p)=e^{-\frac{i \alpha}{2}}\left(\frac{\operatorname{ch}\left(\alpha x+\frac{i \alpha}{2}\right)}{\operatorname{ch}\left(\alpha x-\frac{i \alpha}{2}\right)}\right)^{\frac{1}{2}}\left(\frac{\operatorname{ch}\left(\frac{p}{2}+\frac{i \alpha}{2}\right)}{\operatorname{ch}\left(\frac{p}{2}-\frac{i \alpha}{2}\right)}\right)^{\frac{1}{2}} \mathcal{W}_{-}(x, p) .
\end{aligned}
$$

(Ветвь квадратного корня фиксируется требованием, чтобы соответствующие функции имели предел $e^{i \alpha / 2}$ при $x, p \rightarrow \infty$.) Функция $F_{r}(x, p)$ из раздела 3 работы [3] аналогичным образом связана с волновой функцией $\mathcal{W}_{r}(x, p)$, соответствуюшей спектральным данным

$$
r=i \alpha, \quad \mu(x)=-\frac{e^{i \alpha}}{2 \operatorname{sh}(i \alpha)}, \quad \alpha \in(0, \pi) .
$$

Легко проверить, что (ср. (4.2))

$$
\begin{aligned}
\mathcal{W}_{r}(x, p) & =e^{\frac{\pi p}{2 \alpha}} \mathcal{W}_{+}\left(x+\frac{i \pi}{2} \alpha, p\right)= \\
& =e^{i x p} \frac{e^{i \alpha} \operatorname{ch}\left(\alpha x+\frac{p}{2}\right)-\operatorname{ch}\left(\alpha x-\frac{p}{2}\right)}{2 \operatorname{sh}\left(\alpha x+\frac{i \alpha}{2}\right) \operatorname{sh}\left(\frac{p}{2}+\frac{i \alpha}{2}\right)},
\end{aligned}
$$

и тогда функция $F_{r}$ имеет вид

$$
F_{r}(x, p)=e^{-\frac{i \alpha}{2}}\left(\frac{\operatorname{sh}\left(\alpha x+\frac{i \alpha}{2}\right)}{\operatorname{sh}\left(\alpha x-\frac{i \alpha}{2}\right)}\right)^{\frac{1}{2}}\left(\frac{\operatorname{sh}\left(\frac{p}{2}+\frac{i \alpha}{2}\right)}{\operatorname{sh}\left(\frac{p}{2}-\frac{i \alpha}{2}\right)}\right)^{\frac{1}{2}} \mathcal{W}_{r}(x, p) .
$$

Изменение знака $\mu$ в (4.6) по сравнению со спектральными данными для $\mathcal{W}_{+}(x, p)$ имеет радикальное последствие: преобразование собственных функций, соответствующее $\mathcal{W}_{r}(x, p)$, больше не является изометрическим. С другой стороны, преобразование собственных функций с ядром $(2 \pi)^{-1 / 2} F_{r}(x, p)$ является изометрией из нечетного подпространства в $L^{2}(\mathbb{R}, d p)$ на нечетное подпространство в $L^{2}(\mathbb{R}, d x)$ (см. раздел 3 в работе [3]).

В работе [3] вопросы четности играют основную роль. Приведенные выше унитарные преобразования трех операторов $A_{+}, A_{-}$и $A_{r}$, связанных с $\mathcal{W}_{+}, \mathcal{W}_{-}$и $\mathcal{W}_{r}$, приводят к инвариантным относительно четности $\mathrm{A} \Delta \mathrm{O} H_{a}^{2}-2, H_{e}^{2}+2$ и $H_{r}^{2}-2$ при $N=1$ из работы [3]. Заметим в этой связи, что $\mathrm{A} \Delta \mathrm{O} A$, задаваемые формулой (1.6), явным образом не инвариантны относительно четности (за исключением случая, когда $V_{a}(x)=$ 1 и $V_{b}(x)$ четно).

Чтобы установить связь с результатами работы [3] при произвольном $N$, требуется преобразование унитарного подобия, обобщающее рассмотренное выше такое преобразование для $N=1$. Чтобы задать это преобразование, вспомним формулу (2.7), определяющую $V_{a}(x)$. Из нее следует, что оператор $A$ можно переписать как

$$
A=e^{-i \partial_{x}}+\lambda(x) e^{i \partial_{x}} \lambda(x)^{-1}+V_{b}(x) .
$$


Не очевидно, но верно, что из требований формальной самосопряженности (2.20) и $(2.21)$ следует, что $\lambda^{*}(x)=\lambda(x)^{-1}$. Поэтому $\lambda(x)$ является фазовым множителем при вешественных $x$. В этом случае унитарное подобие имеет вид

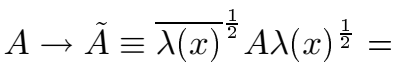

$$
\begin{aligned}
& =\left(\frac{\lambda(x-i)}{\lambda(x)}\right)^{\frac{1}{2}} e^{-i \partial_{x}}+\left(\frac{\lambda(x)}{\lambda(x+i)}\right)^{\frac{1}{2}} e^{i \partial_{x}}+V_{b}(x), \\
& \mathcal{W}(x, p) \rightarrow \widetilde{\mathcal{W}}(x, p) \equiv \overline{\lambda(x)}^{\frac{1}{2}} \mathcal{W}(x, p) \text {. }
\end{aligned}
$$

Квадратные корни выбраны так, чтобы предел $\lambda(x)$ при $x \rightarrow \infty$ был равен единице (см. (2.10)).

Инвариантные относительно четности операторы $H_{a}^{2}-2, H_{e}^{2}+2$ и $H_{r}^{2}-2$ в работе [3] имеют вид (4.7) при произвольном $N$. Но они составляют всего лишш малую часть интересующих нас операторов $\tilde{A}$. Действительно, они соответствуют спектральным данным $r_{1}, \ldots, r_{N}, \mu_{1}, \ldots, \mu_{N}$, которые определены, коль скоро фиксирована величина $\alpha$. Еше одним моментом, заслуживаюшим упоминания, является тот факт, что из соотношений (4.8), (1.9) и (2.10) вытекает, что

$$
\widetilde{\mathcal{W}}(x, p) \sim \begin{cases}e^{i x p}, & \operatorname{Re} x \rightarrow \infty \\ \exp \left(-\sum_{n=1}^{N} r_{n}\right) a(p) e^{i x p}, & \operatorname{Re} x \rightarrow-\infty\end{cases}
$$

Прошедшая волна, таким образом, приобретает дополнительную фазу.

Сказанное выше сушественно и при сравнении со сценарием, предложенным в работе [1]. Взяв за основу результаты работы [3], кажется естественным искать класс безотражательных $\mathrm{A} \Delta \mathrm{O}$ вида

$$
\tilde{A}=V_{+}(x)^{\frac{1}{2}} e^{-i \partial_{x}}+V_{-}(x)^{\frac{1}{2}} e^{i \partial_{x}}+V_{0}(x)
$$

свойства которых указаны в работе [1]. Приведенные здесь результаты можно адаптировать к картине, описанной в [1], однако представленная нами картина имеет определенные отличия. Действительно, если взять за исходный $\mathrm{A} \Delta \mathrm{O} \quad A$, задаваемый формулой (1.6), видим, что этот оператор имеет мероморфные коэффициенты; в этом случае нет точек ветвления, соответствующих квадратным корням, как в операторе $\tilde{A}$. Чтобы получить соответствующие самосопряженные операторы на $L^{2}(\mathbb{R}, d x)$, которые были бы инвариантными относительно четности при подходящих дополнительных условиях, следует допустить унитарное преобразование подобия, включающее квадратные корни из мероморфных функций (см. выше). Возникаюшие в этом весьма специальном случае работы [3] свойства четности могли бы остаться незамеченными при работе с $\mathrm{A} \Delta \mathrm{O}$ вида (1.6). Во всяком случае класс А $\Delta \mathrm{O} \tilde{A}$ вида (4.9), выделенный в работе [1], также заслуживает дальнейшего изучения (заметим, что здесь имеются три a priori независимых потенциала, в отличие от двух потенциалов в (1.6)). 


\section{Список литературы}

[1] S. N. M. Ruijsenaars. J. Nonlin. Math. Phys. (Suppl.). 2001. V. 8. P. 240-248.

[2] S. N. M. Ruijsenaars. J. Math. Phys. 1999. V. 40. P. 1627-1663.

[3] S. N. M. Ruijsenaars. Publ. RIMS Kyoto Univ. 2000. V. 36. P. 707-753.

[4] S. N. M. Ruijsenaars. J. Nonlin. Math. Phys. 2001. V. 8. P. 106-138.

[5] S. N. M. Ruijsenaars. Reflectionless analytic difference operators: II. Relations to soliton systems. J. Nonlin. Math. Phys. (to appear).

[6] S. N. M. Ruijsenaars. Reflectionless analytic difference operators: III. Hilbert space aspects (to appear).

[7] Ф. Калоджеро, А. Дегасперис. Спектральные преобразования и солитоны. Методы решения и исследования нелинейных эволюционных уравнений. М.: Мир, 1985.

[8] A. Нъюэлл. Солитоны в математике и физике. М.: Мир, 1985.

[9] H. Flaschka. Progr. Theor. Phys. 1974. V. 51. P. 703-716.

[10] М. Тода. Теория нелинейных решеток. М.: Мир, 1984.

[11] Л. А. Тахтаджсян, Л. Д. Фаддеев. Гамильтонов подход в теории солитонов. М.: Наука, 1986.

[12] P. M. Santini. Integrable singular integral evolution equations. In: Important Developments in Soliton Theory. Eds. A. S. Fokas and V. E. Zakharov. New York: Springer, 1993. P. 147-177.

[13] S. N. M. Ruijsenaars and H. Schneider. Ann. Phys. 1986. V. 170. P. 370-405.

[14] S. N. M. Ruijsenaars. Publ. RIMS Kyoto Univ. 1994. V. 30. P. 865-1008.

[15] S. N. M. Ruijsenaars. Ann. Phys. 1997. V. 256. P. 226-301. 